\title{
Development of an inactivated COVID-19 vaccine
}

\author{
Kengo SONODA ${ }^{1 *}$ \\ 1 Development Department, R\&D Division, KM Biologics Co., Ltd., 1314-1 Kyokushi Kawabe, Kikuchi-shi, Kumamoto 869-1298, Japan
}

KM Biologics Co., Ltd. (KM Biologics) is a Kumamotobased pharmaceutical company that inherited the major business of the Chemo-Sero-Therapeutic Research Institute (Kaketsuken) in 2018; it started its operation as a consolidated subsidiary of Meiji Holdings Co., Ltd. in July of the same year. The major business segments of KM Biologics are research, development, manufacturing, and supply of human and veterinary vaccines and blood plasma products. Regarding human vaccines, KM Biologics supplies a variety, including influenza, DTaP-IPV, Japanese encephalitis, and hepatitis A and B vaccines, mainly in the domestic market. In response to the outbreak of a novel influenza virus in 2009, we developed a production and distribution system for a pandemic influenza vaccine based on the cell culture method, with the support of the Ministry of Health, Labour and Welfare (MHLW). The system can supply vaccines to immunize 57 million people in half a year. Since the coronavirus disease 2019 (COVID-19) pandemic began in 2020, KM Biologics has been responsible for continuously supplying vaccines in demand, such as influenza and combination vaccines for children, and has also been working on solutions to various COVID-19 issues, one of which is the development of an inactivated vaccine (KD-414).

As of June 2021, there are 102 candidate COVID-19 vaccines in the commercial or clinical trial phase, 16 of which are inactivated vaccines [1]. KD-414 is a liquid inactivated vaccine, containing purified inactivated whole virions of severe acute respiratory syndrome coronavirus 2 (SARSCoV-2) cultured in Vero cells derived from African green monkey kidney cells and aluminum hydroxide as the antigen and adjuvant, respectively. The manufacturing process of KD-414 is fundamentally the same as the well-established process for our commercially approved Japanese encephalitis vaccine, and a similar process was also applied to a prototype West Nile fever inactivated vaccine, a Zika fever inactivated vaccine (JapicCTI-194772) that has completed phase I clinical trial with the support of National Agency for Research and Development (AMED), and a dengue live vaccine, of which Phase II clinical trials are under preparation and a part of the nonclinical efficacy studies were conducted with a grant by the Global Health Innovative Technology (GHIT) Fund. Aluminum hydroxide was selected as the adjuvant of KD-414 considering its stable supply and the

*Correspondence to: Sonoda, K.: sonoda-ke@kmbiologics.com Received: Sep. 6, 2021; Accepted: Nov. 15, 2021 extensive research available with the commercial vaccine. The storage temperature is planned to be $2^{\circ} \mathrm{C}$ to $8^{\circ} \mathrm{C}$, and we expect to make full use of the above-mentioned distribution system for prompt supply. The development of KD-414 requires handling of SARS-CoV-2 at a biosafety level of 3 , and in this regard, we have well-controlled facilities, accumulated technologies, and experienced personnel in terms of biosafety and biosecurity. For future large-scale production, the pilot (600 1 and production plant $(4,5001 \times$ 3 units) previously built for the aforementioned influenza vaccine are being remodeled with the support of the project entitled "Project of construction for vaccine production system in FY2020", and actual production is planned to start in the spring of 2022. The initial development plan was adopted by the "R\&D on the novel coronavirus disease (COVID-19)" by AMED in May 2020. With the funding, activities for development such as drug efficacy studies are progressing in collaboration with Dr. Hideki Hasegawa, Director of Influenza Vaccine Research Center at the National Institute of Infectious Diseases (NIID), Professor Yoshihiro Kawaoka and Associate Professor Masaki Imai of the Institute of Medical Science at The University of Tokyo (IMSUT), and Yumiko Imai, Project Leader of the National Institutes of Biomedical and Innovation, Health and Nutrition (NIBIOHN)(The job titles and affiliations are listed as they were at that time). Furthermore, the late development plan was adopted by the second term of "R\&D on the novel coronavirus infectious disease (COVID-19)" by AMED in October 2020, and the scale-up of manufacturing methods, manufacturing of next-phase investigational products, and establishment of quality control tests are ongoing.

The development of KD-414 began with the abovementioned grants by AMED in May 2020. The production of the experimental vaccine was completed, and successful induction of neutralizing antibodies against SARS-CoV-2 was confirmed in mice by July 2020. In August 2020, a repeated dose toxicity study in rabbits was initiated as a nonclinical safety study, and the results showed no issue proceeding to clinical studies. We also obtained nonclinical efficacy data in mouse models at NIID and hamster models at IMSUT. In addition, a nonclinical efficacy study in African green monkey models is currently ongoing, and it has been confirmed that 160 to 640 times more the number of neutralizing antibodies against SARS-CoV-2 were induced with two administrations of KD-414 at four-week intervals. The efficacy against SARS-CoV-2 variants was evaluated using 
sera from mice immunized with KD-414, and a comparable level of neutralizing activity was confirmed against the $\alpha$, $\beta, \gamma$, and $\delta$ variants. In the future, a similar assessment will be conducted using sera obtained from participants in a phase I/II clinical trial. Since March 2021, a multicenter, double-blind, randomized, parallel-group comparative study with a total of 210 healthy adults aged between 20 and 65 years and over 65 years (jRCT2071200106) has been ongoing. After two administrations at four-weeks intervals, no serious adverse events, such as anaphylaxis, have been reported, and no particular safety concerns have been raised (type and rate of reported adverse events are comparable to those commonly observed in inactivated vaccines). Immunogenicity is currently being evaluated, and the results, including neutralizing antibody titers against SARS-CoV-2 and binding antibody titers against the $S$ protein of the virus, will be summarized in the future.

The efficacy of the COVID-19 vaccines that have been approved in Japan has been evaluated through largescale, placebo-controlled efficacy studies. However, there are ethical issues in conducting such trials in the current situation wherein multiple vaccines are already in use. Therefore, the design is under consideration in accordance with the guidelines in "The idea of rebalancing" by the
Pharmaceuticals and Medical Devices Agency (PMDA); that is, we plan to gather alternative data from efficacy studies in appropriate animal models, clinical safety studies on an appropriate scale, active-controlled immunogenicity studies, and post-marketing efficacy studies and surveillance to provide evidence relevant to placebo-controlled studies.

Currently, the use of mRNA vaccines is advanced in Japan. However, side reactions such as fever and fatigue after the second dose have been reported at a high frequency. With the safety and efficacy being proven in future clinical studies, an inactivated COVID-19 vaccine, KD-414, would be a safer choice and could prompt the willingness of the people to take COVID-19 vaccines.

\section{Potential Conflicts of Interest}

The authors have nothing to disclose.

\section{Reference}

1. COVID-19 vaccine tracker and landscape, https://www. who.int/publications/m/item/draft-landscape-of-covid-19candidate-vaccines [accessed June 1, 2021]. 